\title{
Electron and hole confinement in stacked self-assembled InP quantum dots
}

\author{
M. Hayne and R. Provoost \\ Laboratorium voor Vaste-Stoffysica en Magnetisme, Katholieke Universiteit Leuven, Celestijnenlaan 200D, B-3001 Leuven, Belgium \\ M. K. Zundel, Y. M. Manz, and K. Eberl \\ Max-Planck-Institut für Festkörperforschung, Heisenbergstrasse 1, 70569 Stutttgart, Germany \\ V. V. Moshchalkov \\ Laboratorium voor Vaste-Stoffysica en Magnetisme, Katholieke Universiteit Leuven, Celestijnenlaan 200D, B-3001 Leuven, Belgium
}

(Received 3 April 2000)

\begin{abstract}
We report photoluminescence measurements on stacked self-assembled InP quantum dots in magnetic fields up to $50 \mathrm{~T}$. For triply stacked layers the dots become strongly coupled when the layer separation is $4 \mathrm{~nm}$ or less. In contrast, doubly stacked layers show no sign of coupling. We explain this puzzling difference in coupling by proposing a model in which the holes are weakly confined in the $\mathrm{Ga}_{x} \mathrm{In}_{1-x} \mathrm{P}$ layers separating the layers of dots, and are responsible for the coupling. Since only one such intervening layer exists in the doubly stacked dots coupling is excluded. Our model is strongly supported by the exciton masses and radii derived from our experimental results, and is consistent with available theory.
\end{abstract}

\section{INTRODUCTION}

The properties of self-assembled quantum dots are currently the subject of intense investigation. ${ }^{1}$ Their appeal lies in the attractive combination of fascinating physics with very strong potential for applications such as lasers. ${ }^{2}$ Despite this activity, many of the basic properties of these structures are not well understood. This is a direct result of some of the very qualities which make them interesting: namely their small size $(\sim 10 \mathrm{~nm})$ and the self-assembling process by which they are grown. Both of these advantages also limit our ability to investigate and control their properties.

Thus the growth of self-assembled quantum dots by the Stranski-Krastonow mode presents some interesting dilemmas. The self-assembling process allows the production of billions of highly uniform dots per $\mathrm{cm}^{2}$ of semiconductor wafer in a single technological step. Yet because the dots are self-assembling, our ability to control their growth is limited. One way of asserting control over the properties of the dots is to introduce several further degrees of freedom by growing them in stacked layers. In these structures the number of layers, their separation, and the amount of material in each layer can be carefully tuned. The strain relaxation mechanism which causes the self-assembling process is also exploited in the growth of vertically aligned stacks of selfassembled quantum dots.

During the Stranski-Krastanow growth mode the deposition of a few monolayers of a semiconductor alloy such as InAs or InP on a lattice-mismatched substrate (usually GaAs or $\mathrm{Ga}_{x} \mathrm{In}_{1-x} \mathrm{P}$, respectively) results in the spontaneous formation of nanometer sized droplets, thereby reducing the strain energy compared with a continuous uniform film of material. When further layers of substrate material are deposited on top of the dots they have a nonuniform strain distribution: The lattice is dilated at the positions where the underlying dots are located. Provided that the thickness of the intervening layer of substrate material is not so thick that the strain distribution is relaxed at the surface, the further deposition of dot material results in the formation of new dots at these preferential sites. This is shown schematically in Fig. 1. Using this technique large arrays of self-aligning stacks of self-assembled quantum dots can be grown, but with the advantage over single layers that the layer separation, number, and even dot size can be varied. One interesting consequence of this type of structure is the ability to study the effects of coupling between the dots, and eventually to control its strength. This was the subject of a previous report in which we demonstrated the observation of strong electronic coupling in triply stacked layers of InP self-assembled quantum separated by $4 \mathrm{~nm}$ or less. ${ }^{3}$ Here we shall review these results, and by comparing them with new data on doubly stacked layers show that the coupling can be attributed to the holes, which are weakly confined in the highly strained $\mathrm{Ga}_{x} \mathrm{In}_{1-x} \mathrm{P}$ separating the dots. We shall go on to show how our results can be used to build up a detailed picture of the electron and hole confinement in stacked layers of selfassembled InP quantum dots.

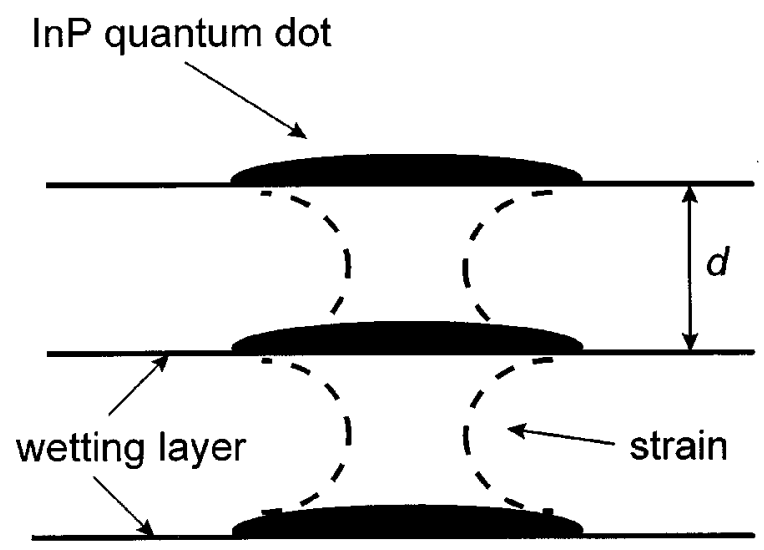

FIG. 1. Schematic diagram showing the structure of the triply stacked InP quantum dot samples studied here. The dots sit in a $\mathrm{Ga}_{0.52} \mathrm{In}_{0.48} \mathrm{P}$ matrix. The strain between the layers of dots which is responsible for the stacking mechanism is represented by the dotted lines (schematic). 


\section{QUANTUM DOTS IN A MAGNETIC FIELD}

A particularly useful technique for the study of selfassembled quantum dots is a strong magnetic field $B$. At zero and low magnetic fields the electron (and hole) within the dot are strongly spatially confined by the physical boundaries of the dot. In this regime the applied field makes only a small perturbation and (neglecting spin) the electron and hole states in the dots increase in energy according to $\Delta E_{e, h}$ $=\left\langle\rho_{e, h}^{2}\right\rangle B^{2} / 8 m_{e, h}$, where $\sqrt{\left\langle\rho_{i}^{2}\right\rangle}$ and $m_{i}$ are the electron (hole) effective radius and mass in the plane perpendicular to $B{ }^{4}$ At sufficiently high field, when the attempted Larmor radius is smaller than the spatial size of the dot, the charges become confined by the field in the plane perpendicular to the direction in which it is applied, and the energy levels shift linearly with $B$, as they do for a two-dimensional system. In this limit the energy shift depends only on the effective mass, thus both the effective mass and radius can be determined. The very small size of self-assembled quantum dots makes achieving the high-field limit extremely difficult in a conventional superconducting magnet, but such fields are achievable using pulsed magnets.

For the analysis of experimental data a simple function can readily be constructed which behaves according to the above description in the appropriate limits. Furthermore, by requiring that the function and its derivatives are continuous at the boundary between the two regimes we remove the need for any additional unknown parameters. Thus we obtain

$$
\begin{gathered}
E_{\mathrm{CM}}=E_{\mathrm{CM}}^{0}+\frac{e^{2}\left\langle\rho^{2}\right\rangle}{8 \mu} B^{2} \quad \text { for } B<\frac{2 \hbar}{e\left\langle\rho^{2}\right\rangle}, \\
E_{\mathrm{CM}}=E_{\mathrm{CM}}^{0}-\frac{\hbar^{2}}{2 \mu\left\langle\rho^{2}\right\rangle}+\frac{\hbar e B}{2 \mu} \text { for } B>\frac{2 \hbar}{e\left\langle\rho^{2}\right\rangle} .
\end{gathered}
$$

We have parametrized the average energy of the large ensemble of dots probed in the experiment as $E_{\mathrm{CM}}$ the center of mass of the photoluminescence (PL) peak. This can be obtained from the experimental results with a very high degree of accuracy, as can be seen from the scatter in the data points in Figs. 2 and 3. $E_{\mathrm{CM}}^{0}$ is the position of the center of mass at $B=0$. Note that we have followed the conventional approach by combining the influence of magnetic field on the electron and hole levels by introducing the exciton effective mass $\mu$ and effective exciton radius $\sqrt{\left\langle\rho^{2}\right\rangle}$. This implicitly assumes that the electron and hole radii are the same, an assumption which our results shall later show cannot be generally justified. Finally, we point out that the field at which Eq. (1) goes from the low-field to the high-field limit corresponds to the point where the magnetic length is equal to $1 / \sqrt{2}$ times the exciton radius.

\section{EXPERIMENTAL DETAILS}

The self-assembled InP quantum dot samples were grown by solid source molecular beam epitaxy. Nominally 3.0 ML of InP was deposited on $50 \mathrm{~nm}$ of $\mathrm{Ga}_{0.52} \mathrm{In}_{0.48} \mathrm{P}$, followed by $\mathrm{Ga}_{0.52} \mathrm{In}_{0.48} \mathrm{P}$ spacer layers of nominal thickness $d$. Samples $B, C$, and $D$ contained triply stacked layers of dots with $d$ $=8,4$, and $2 \mathrm{~nm}$, respectively (Fig. 1). Sample $E$ contained two stacked layers with $d=4 \mathrm{~nm}$, while sample $A$ contained only a single layer of dots. The samples were all capped with

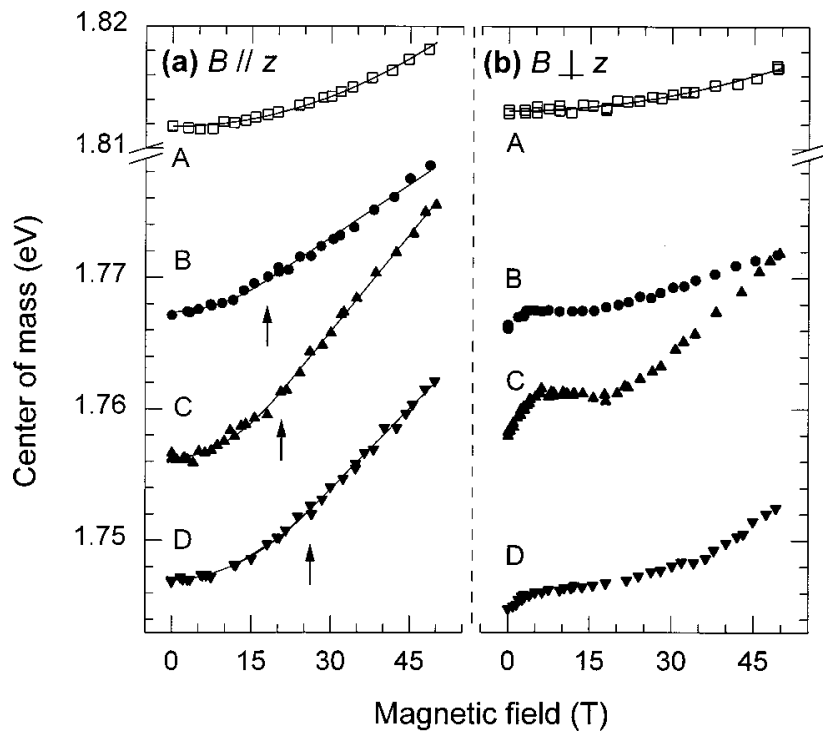

FIG. 2. $E_{\mathrm{CM}}$ with $B$ applied (a) parallel (\|) and (b) perpendicular $(\perp)$ to the growth direction $(z)$ for samples $A-D$. The solid lines are fits to Eq. (1), except for sample $A$ where the fit is parabolic up to the highest fields. The arrows indicate the crossover from low to high field dependence as determined by the fits. For these data entirely parabolic fits (not shown) are rather poor.

a further layer of $\mathrm{Ga}_{0.52} \mathrm{In}_{0.48} \mathrm{P}$. Transmission electron microscopy (TEM) studies showed the dots to be lens or disc shaped with approximately $16 \mathrm{~nm}$ diameter and $2 \mathrm{~nm}$ height, and that the dots in the stacked-layer samples were nicely vertically aligned. Full details of the growth procedure including TEM images can be found in Ref. 5.

The PL experiments were carried out at $4.2 \mathrm{~K}$ in a He bath cryostat placed in the bore of a pulsed magnet with a maximum field of $50 \mathrm{~T}$. The field was applied perpendicular and parallel to the growth direction $(z)$. A bundle of six $400-\mu \mathrm{m}$ core optical fibers were used to collect the PL, which was excited by the light from a frequency-doubled solid-state laser $(20 \mathrm{~mW}$ at $532 \mathrm{~nm})$ via a seventh fiber in the center of the bundle. The PL was dispersed in a $0.275 \mathrm{~m}$

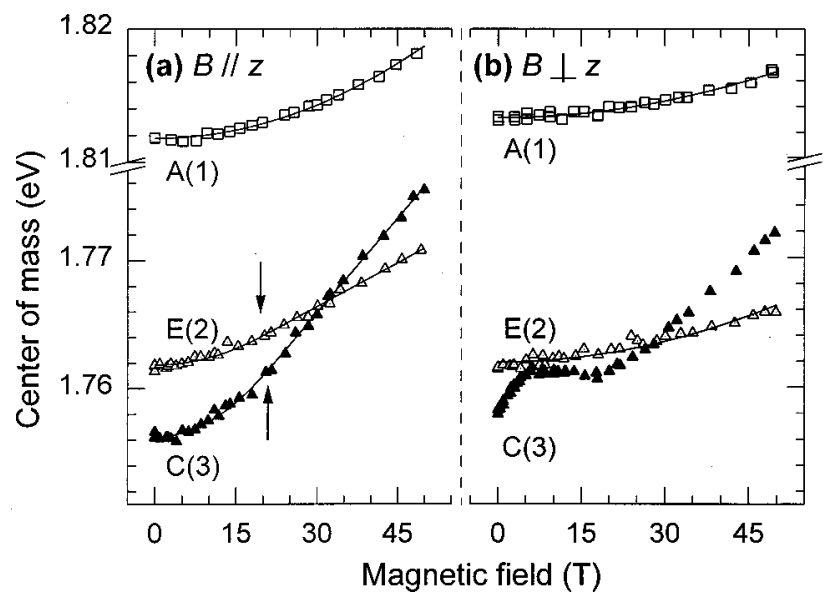

FIG. 3. As for Fig. 2 but for the double layer sample $E$. The results for samples $A$ and $C$ are also shown for comparison. The numbers in brackets refer to the number of layers of InP dots in the sample. 
focal length spectrometer and detected by an intensified charge-coupled-device camera. By measuring the PL at the peak of field pulse a field resolution of $\pm 1 \%$ was achieved with an integration time of $1.8 \mathrm{~ms}$. Additional data were obtained during the down sweep of the field with a resolution of $\pm 3 \%$ and an integration time of $0.3 \mathrm{~ms}$.

\section{EXPERIMENTAL RESULTS}

The observation of strong electronic coupling in triply stacked layers of self-assembled InP quantum dots has already been described elsewhere. ${ }^{3}$ The main results of this investigation are reproduced in Fig. 2. We observe three separate pieces of evidence for the onset of strong electronic coupling when the layer separation is $4 \mathrm{~nm}$ or less. These are (i) A large reduction in PL linewidth for closely stacked samples. This is a widely reported effect, and is attributed to the reduction in the influence of fluctuations in the height of the dots once they become coupled in a stack. ${ }^{3,5,6}$ Prior to our investigation this was the only reported evidence for quantum-mechanical coupling in stacked self-assembled quantum dots. (ii) With the field applied perpendicular to $z$ the shift of $E_{\mathrm{CM}}$ is a measure of the confinement in the growth direction. A large increase in the size of the shift of $E_{\mathrm{CM}}$, from $5.5 \mathrm{meV}$ in sample $B$ to $13.8 \mathrm{meV}$ in sample $C$, indicates a strong reduction in the spatial confinement in the growth direction, and a change from confinement by a single dot in this direction to confinement by the entire stack. Consistent with this explanation is the observation that for sample $D$, where the layer separation is reduced to $2 \mathrm{~nm}$ and the stack height is correspondingly smaller, the shift is also reduced, to $7.7 \mathrm{meV}$. By the same argument, the small shift in sample $B$, which has the largest stack height, indicates that the dots are not coupled. (iii) When the field is applied perpendicular to $z$, the field dependence of $E_{\mathrm{CM}}$ shows anomalous behavior in the stacked layer samples $C$ and $D$, inconsistent with the behavior predicted by Eq. (1). This behavior is not understood, and will not be discussed here. For the present we shall assert that since such behavior has never been observed in any studies of single quantum dots (including our own), its explanation requires some complex behavior which is linked to the fact that the dots are coupled. There is also a hint of the same behavior in sample $B$ at very low fields. This may indicate that a small proportion of the dots are weakly coupled at low fields, but that the coupling is destroyed by applying a magnetic field in the plane of the layers. Note that the magnetic length is equal to the layer separation in this sample at $10 \mathrm{~T}$, whereas for samples $C$ and

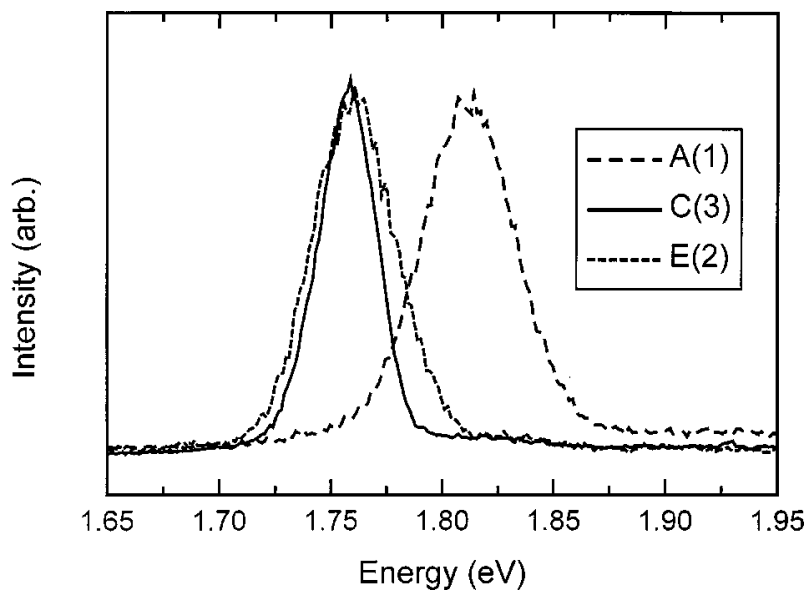

FIG. 4. Zero-field spectra for samples $A, C$, and E. The data were taken in $1.8 \mathrm{~ms}$ at $4.2 \mathrm{~K}$.

$D$ this limit is reached at 40 and 160 T, respectively.

The study of triply stacked layers of InP quantum dots has revealed strong quantum-mechanical coupling for $d \leqslant 4 \mathrm{~nm} .^{3}$ For the remainder of this report we shall go on to demonstrate that reducing the number of stacked layers from three to two has allowed us to build up a detailed and consistent picture of the electron and hole confinement in stacked selfassembled InP quantum dots. Sample $E$ is a double-layer sample with $d=4 \mathrm{~nm}$. This value was chosen because the results for the equivalent triple-layer sample were the most striking. A double-layer sample with $d=2 \mathrm{~nm}$ was also studied, but will not be discussed in detail here. The results from this sample are consistent with our interpretation, but not as clear due to a combination of the small stack height $(5.4 \mathrm{~nm})$ and some unintentional differences in the growth compared with the other samples.

Figure 3 presents the field dependence of $E_{\mathrm{CM}}$ for sample $E$. The results for samples $A$ and $C$ are also given for comparison. First we discuss the points of similarity between samples $C$ and $E$. Figure 2 shows that $E_{\mathrm{CM}}^{0}$ systematically moves towards lower energy as $d$ is reduced. This is due to the effects of strain relaxation in the dots, with some additional contribution from the effects of coupling. ${ }^{3,5}$ Figures 3 and 4 show that $E_{\mathrm{CM}}^{0}$ for samples $C$ and $E$ are close, implying that the strain distribution in the two samples are very similar. In addition, the electronic size of the quantum dots (Table I) as measured by the transition from the low- to high-field regime (indicated by the arrow in Fig. 3) is the same. We therefore conclude that the dots in both samples are structurally the same; they have similar strain distribu-

TABLE I. Summary of the sample parameters and experimental data. The effective masses and radii are determined by a fit of the data to Eq. (1).

\begin{tabular}{cccccccc}
\hline \hline $\begin{array}{c}\text { Sample, } \\
\text { (\# layers) }\end{array}$ & $\begin{array}{c}\text { Layer } \\
\text { separation } \\
(\mathrm{nm})\end{array}$ & $\begin{array}{c}\text { Stack } \\
\text { height } \\
(\mathrm{nm})\end{array}$ & $\begin{array}{c}\mathrm{PL} \\
\text { linewidth } \\
(\mathrm{meV})\end{array}$ & $\begin{array}{c}\text { Shift of } E_{\mathrm{CM}} \\
(B \| z) \\
(\mathrm{meV})\end{array}$ & $\mu\left(m_{0}\right)$ & $\begin{array}{r}\left\langle\rho^{2}\right\rangle \\
(\mathrm{nm})\end{array}$ & $\begin{array}{c}\text { Shift of } E_{\mathrm{CM}} \\
(B \perp z) \\
(\mathrm{meV})\end{array}$ \\
\hline$A(1)$ & & & 43 & 6.3 & & & 3.6 \\
$B(3)$ & 8 & 20 & 40 & 11.4 & 0.22 & 8.6 & 5.5 \\
$C(3)$ & 4 & 12 & 28 & 19.4 & 0.12 & 8.0 & 13.8 \\
$D(3)$ & 2 & 8.3 & 24 & 15.2 & 0.14 & 7.1 & 7.7 \\
$E(2)$ & 4 & 7.4 & 37 & 9.4 & 0.25 & 8.1 & 4.5 \\
\hline \hline
\end{tabular}




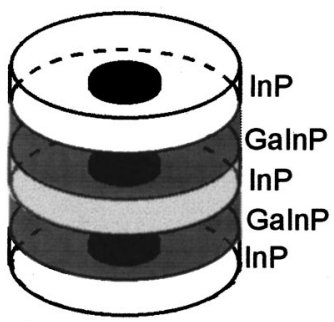

(a)

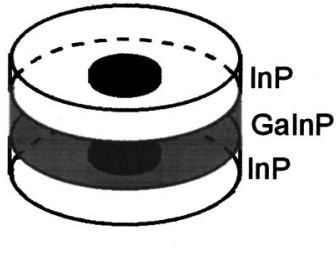

(b)
FIG. 5. Schematic representation of the proposed electron and hole wave function distributions in (a) triply and (b) doubly stacked InP quantum dots. The dark shaded regions indicate the electrons, which are thought to be tightly confined to the center of the InP dots. The lighter and lightest shaded regions indicate the hole wave function distribution and how they couple.

tions and have the same electronic size. In all other aspects the results for sample $E$ look remarkably like those for sample $A$, which has only a single layer of dots. We believe that this is because the dots in sample $E$ are uncoupled. Consider the three pieces of evidence for coupling in the triply stacked layers. These were (i) a large reduction in linewidth, (ii) reduced confinement in the growth direction (as indicated by the shift of $E_{\mathrm{CM}}$ with $B$ applied in the plane of the layers), and (iii) an anomalous field dependence with $B$ applied in the plane of the layers. Sample $E$ shows none of these. The linewidth is slightly reduced compared to samples $A$ and $B$, but still much larger than samples $C$ and $D$ (Fig. 4, Table I). Also the shift of $E_{\mathrm{CM}}$ with $B$ applied in the plane of the layers shows no sign of the anomalous behavior of the coupled samples, and it is very small $(4.5 \mathrm{meV})$. The small blueshift of sample $E$ compared to sample $C$ is also consistent with a loss of coupling. Clearly, by changing from three stacked layers of dots to two we have destroyed the coupling between the dots.

In order to explain this startling result we propose a model for the confinement in stacked self-assembled InP quantum dots in which the holes are weakly confined in the highly strained $\mathrm{Ga}_{x} \mathrm{In}_{1-x} \mathrm{P}$ layers separating the dots, and are responsible for the coupling. Calculations for single pyramidical InP quantum dots ${ }^{7}$ show that the electrons are tightly bound in the center of the dots, whereas the holes are confined in a broken ring in the strained $\mathrm{Ga}_{x} \mathrm{In}_{1-x} \mathrm{P}$ material around the edge of the dots. Note that for InP dots in $\mathrm{Ga}_{x} \mathrm{In}_{1-x} \mathrm{P}$ the valence band offset is negative, i.e., the dots are type II. The holes are confined entirely by the effects of strain. In the case of stacked layers we know that the stacking mechanism is the result of the strained $\mathrm{Ga}_{x} \mathrm{In}_{1-x} \mathrm{P}$ material separating the layers of dots providing preferential sites for the nucleation of new dots (Fig. 1). These new dots will further strain the $\mathrm{Ga}_{x} \mathrm{In}_{1-x} \mathrm{P}$, making it a prime site for the confinement of the holes, which will therefore be confined in-between the layers of dots. This is shown schematically in Fig. 5. Indeed, even for InAs dots, which are normally type I, a transition to type II behavior is predicted for sufficiently closely stacked dots, also as a result of strain. ${ }^{8}$ With this model we can now readily explain why the triply stacked dots couple, but the doubly stacked dots do not. The triply stacked dots have two regions of $\mathrm{Ga}_{x} \mathrm{In}_{1-x} \mathrm{P}$ separating them, thus coupling can occur via the holes. In contrast the doubly stacked layers have only one such layer, and hole coupling is excluded. The electrons do not couple. The large hole effective mass usually makes it an unlikely candidate for coupling, but here this is more than offset by the weakness of the hole confinement and the strength of the electron confinement by large band offsets. If the electrons were to couple we should expect to see the effect of this in sample $E$. In the following section we shall demonstrate that a close examination of the experimental data for all the samples strongly supports our model, and justifies the schematic wavefunction distributions of Fig. 5.

\section{DISCUSSION}

We have shown that the strong coupling observed in triply stacked InP quantum dots is absent when only two stacked layers of dots are present. We identified the holes, which are confined by strain in the $\mathrm{Ga}_{x} \mathrm{In}_{1-x} \mathrm{P}$ layers between the dots, as playing the key role in the coupling. Here we shall make a detailed examination of the effective masses and radii obtained for the dots with $B$ applied in the growth direction to support our model.

We first turn to the effective masses. Measurements of exciton masses in $\mathrm{In}_{0.5} \mathrm{Ga}_{0.5}$ As dots grown on $311 \mathrm{~A}$ oriented GaAs substrates found values of $0.050 m_{0}$ in the dots and $0.046 m_{0}$ in the wetting layer, which are reasonable values for such structures. ${ }^{9}$ (The electron mass in $\operatorname{In}_{0.47} \mathrm{Ga}_{0.53} \mathrm{As}$ is $0.05 m_{0} \cdot{ }^{10}$ ) In our samples the effective masses, which are determined by a fit of the data to Eq. (1), range from $0.12 m_{0}$ for sample $C$ to $0.25 m_{0}$ for sample $E$, and in all cases they are much larger than the exciton effective mass for bulk InP $\left(0.0678 m_{0}\right)$. Enhanced exciton effective masses are expected as a result of strain in the dots, but increases by more than 3.5 from the bulk value are difficult to justify on these grounds. Furthermore, the greatest difference in mass is between samples $C$ and $E$, the two samples we have just argued have similar strain distributions. There are three other possible explanations for this large mass; first that the movement of the PL line is dominated by the holes, second that the electron wave function extends out of the InP dots, or third that there is a significant amount of $\mathrm{Ga}$ in the InP dots themselves. (The bulk $\mathrm{Ga}_{x} \mathrm{In}_{1-x} \mathrm{P}$ exciton mass in our samples is $0.2 m_{0}$.) The second explanation may be immediately discounted, since the measured exciton radius is found to be equal to or less than the dot radius as measured by TEM. The third explanation cannot be so easily ruled out, however a careful examination of the experimental data points towards the first explanation.

As discussed in the introduction, it is common practice to combine the field dependence of the electron and hole energy levels when considering the field dependence of the PL energy. Implicit in this approach is the assumption that the electron and hole wave functions occupy the same area perpendicular to the magnetic field, i.e., $\sqrt{\left\langle\rho_{e}^{2}\right\rangle}=\sqrt{\left\langle\rho_{h}^{2}\right\rangle}$. This assumption is probably reasonable for type I dots, such as InAs in GaAs. Consider the case where these quantities are far from equal, in particular where $\sqrt{\left\langle\rho_{e}^{2}\right\rangle} \ll \sqrt{\left\langle\rho_{h}^{2}\right\rangle}$. In this case the holes will reach the high field regime before the electrons, and the effective mass measured from the slope of the PL line will be that of the holes. Even with a relatively modest difference in the wave-function areas (e.g., a factor of 2), the electrons may remain in the low-field regime. The 
large effective masses which we obtain are consistent with this being the case for our samples.

Further evidence in favor of a large hole radius and a small electron radius can be found in the behavior of the single layer sample $A$. In contrast to the other samples, the shift of the PL line remains parabolic up to the highest fields, i.e., the high-field regime is never reached. If the electron radius in the InP dots was similar to the dot radius, then this sample should also reach the high-field limit. On the other hand, the fact that the holes also fail to reach the high-field limit is consistent with our model, and in particular with the theoretical expectations. ${ }^{7}$ In this sample there is only a single layer of dots, and no $\mathrm{Ga}_{x} \mathrm{In}_{1-x} \mathrm{P}$ separating stacked layers of dots as in the other samples. Therefore, rather than the form of the wave functions shown in Fig. 5, we should return to the theory of Prior, Pistol, and Samuelson, ${ }^{7}$ in which the hole wave functions are expected to form a (broken) ring. In this situation the length scale which defines the transition from low- to high-field behavior will not be the diameter of the ring, but its cross-sectional thickness in the plane of the layers. This will happen at very high fields. With this in mind it is interesting to imagine what effect the change in strain has on the hole confinement when the stacked layers of dots come closer and closer. When the layers of dots are well separated but still strongly mechanically strain coupled (vertically aligned) we expect the hole wave function to form a disc, as depicted in Fig. 5. For layers which are so close that they form a single layer, we would expect the hole wave function to form a ring. The most plausible way to get from one limit to the other is to imagine a small opening in the center of the disclike hole wave function which grows in size as the layers become closer and closer. Table I shows that there is a clear trend towards smaller (hole) wave-function radii as the layer separation is reduced. According to the above description this is not a result of a shrinkage in the outer diameter of the hole wave function, but due to the increase in the size of the opening in the middle.

Before concluding we briefly return to the effective masses. Examining the results in more detail reveals a strik- ing correlation. The two samples with coupled quantum dots $(C$ and $D$ ) have almost the same effective mass, as do the samples with uncoupled dots $(B$ and $E$ ), but with a factor of 2 difference between coupled and uncoupled samples. This can also be understood with reference to Fig. 5. For the uncoupled samples the holes are constrained entirely within the $\mathrm{Ga}_{x} \mathrm{In}_{1-x} \mathrm{P}$, but for the coupled samples the hole wave function penetrates into the InP. Exciton effective masses for $\mathrm{Ga}_{x} \mathrm{In}_{1-x} \mathrm{P}$ can vary depending on the ordering within the sample, ${ }^{11}$ but are generally rather high, and certainly higher than for InP. (For our samples we obtain a value of $0.2 m_{0}$ from the field dependence of the $\mathrm{Ga}_{x} \mathrm{In}_{1-x} \mathrm{P}$ peak.) Thus the systematically lower masses in the coupled samples are consistent with a spread of the hole wave function from the $\mathrm{Ga}_{x} \mathrm{In}_{1-x} \mathrm{P}$ into the InP. Indeed, such behavior discounts the possibility of electron coupling between the dots. If this were to be the case we should see the opposite behavior, i.e., increased electron wave-function penetration from the InP into the $\mathrm{Ga}_{x} \mathrm{In}_{1-x} \mathrm{P}$ for coupled dots would give rise to an increased exciton mass.

\section{CONCLUSIONS}

We have studied the photoluminescence of stacked layers of self-assembled InP quantum dots in magnetic fields up to $50 \mathrm{~T}$. The strong quantum-mechanical coupling which is observed in closely stacked triple-layer samples is absent when there are only two stacked layers. We explain this using a model in which the holes are weakly bound in the $\mathrm{Ga}_{x} \mathrm{In}_{1-x} \mathrm{P}$ material separating the dots and are responsible for the coupling. Measurements of effective mass and radii derived from the field dependence of the photoluminescence strongly support this picture.

\section{ACKNOWLEDGMENTS}

This work was supported by the FWO-Vlaanderen, the Flemish Institute for the Promotion of ScientificTechnological Research in the Industry the Flemish GOA, and the Belgian IUAP programmes.
${ }^{1}$ F. Boscherini, G. Capellini, L. Di Gaspare, F. Rosei, N. Motta, and S. Mobilio, Appl. Phys. Lett. 76, 682 (2000); M. Strassburg, Th. Deniozou, A. Hoffman, R. Heitz, U. W. Pohl, D. Bimberg, D. Litvinov, A. Rosenauer, D. Gerthsen, S. Schwedhelm, K. Lischka, and D. Schikora, ibid. 76, 685 (2000); T. M. Hsu, Y. S. Lan, W.-H. Chang, N. T. Yeh, and J.-I. Chyi, ibid. 76, 691 (2000); H. Htoon, J. W. Keto, O. Baklenov, A. L. Holmes, Jr., and C. K. Shih, ibid. 76, 700 (2000).

${ }^{2}$ Y. M. Manz, O. G. Schmidt, and K. Eberl, Appl. Phys. Lett. 76, 3343 (2000); P. W. Fry, L. Harris, S. R. Parnell, J. J. Finley, A. D. Ashmore, D. J. Mowbray, M. S. Skolnick, G. Hill, and J. C. Clark, J. Appl. Phys. 87, 615 (2000); F. Heinrichsdorff, Ch. Ribbat, M. Grundmann, and D. Bimberg, Appl. Phys. Lett. 76, 556 (2000).

${ }^{3}$ R. Provoost, M. Hayne, V. V. Moshchalkov, M. K. Zundel, and K. Eberl, Appl. Phys. Lett. 75, 799 (1999).

${ }^{4}$ P. D. Wang, J. L. Merz, S. Fafard, R. Leon, D. Leonard, G. Mederios-Ribeiro, P. M. Petroff, K. Uchida, N. Muira, H.
Akiyama, and H. Sakaki, Phys. Rev. B 53, 16458 (1996).

${ }^{5}$ M. K. Zundel, P. Specht, K. Eberl, N. Y. Jin-Phillipp, and F. Phillipp, Appl. Phys. Lett. 71, 2972 (1997).

${ }^{6}$ G. S. Solomon, J. A. Trezza, A. F. Marshall, and J. S. Harris, Jr., Phys. Rev. Lett. 76, 952 (1996); R. Heitz, A. Kalburge, Q. Xie, M. Grundmann, A. Hoffman, A. Madhukar, and D. Bimberg, Phys. Rev. B 57, 9050 (1998).

${ }^{7}$ C. Pryor, M-E. Pistol, and L. Samuelson, Phys. Rev. B 56, 10404 (1997).

${ }^{8}$ C. Pryor, Phys. Rev. Lett. 80, 3579 (1998).

${ }^{9}$ R. K. Hayden, K. Uchida, N. Miura, A. Polimeni, S. T. Stoddart, M. Henini, L. Eaves, and P. C. Main, Physica B 249-251, 262 (1998).

${ }^{10}$ A. Kastalsky, R. Dingle, K. Y. Cheng, and A. Y. Cho, Appl. Phys. Lett. 41, 274 (1982).

${ }^{11}$ P. Ernst, Y. Zang, F. A. J. M. Driessen, A. Mascarenhas, E. D. Jones, G. Geng, F. Scholz, and H. Schweizer, J. Appl. Phys. 81, 2814 (1997). 